\title{
Dynamics of The Tranquil Cosmic Web
}

\author{
Adi Nusser \\ Technion- Israel Institute of Technology \\ 32000 Haifa, Israel \\ email: adi@physics.technion.ac.il
}

\begin{abstract}
The phase space distribution of matter out to $\sim 100 \mathrm{Mpc}$ is probed by two types of observational data: galaxy redshift surveys and peculiar motions of galaxies. Important information on the process of structure formation and deviations from standard gravity have been extracted from the accumulating data. The remarkably simple Zel'dovich approximation is the basis for much of our insight into the dynamics of structure formation and the development of data analyses methods. Progress in the methodology and some recent results is reviewed.
\end{abstract}

Keywords. cosmology: large-scale structure of universe

\section{Introduction}

Merging and star formation activities in the galaxy population have calmed gown by the current epoch $(z=0)$. This lead to the establishment of $a)$ a tight relation between the distributions of galaxies and the underlying mass of the dark matter, and $b$ ) relations between galaxy intrinsic properties, allowing for measurements of distance. Therefore, the $z \sim 0$ Large Scale Structure is an excellent laboratory for probing cosmological models. Two complementary observational sets are our main window to the phase space distribution of matter. The first, surveys of galaxy redshifts, $c z$, and apparent magnitudes, $m$. The second, distance measurements $d_{e}$, and hence peculiar motions $v_{p}$, of galaxies obtained via intrinsic relations such as the Tully-Fisher (TF). Distance measurements are more difficult to obtain than just $c z$ and $m$ and hence the number of galaxies with observed peculiar motions is significantly smaller than in redshift surveys. An example of the first set is Two Micron All Sky Redshift Survey (2MRS) (Huchra et al. 2012), of about 45000 galaxies with a mean redshift of $\sim 8000 \mathrm{kms}^{-1}$ and the deeper SDSS containing about half a million galaxies but with partial sky coverage. The second type of data include the SFI++ catalog (Masters et al. 2006) of TF measurements of $\sim 4000$ galaxies, and the Cosmic Flows 2 (CF2)(Tully et al. 2013) catalog of $\sim 8000$. Fig. 1 is a visual representation of the data in the Super-galactic $(\mathrm{SG})$ plane. Note the patchiness and sparseness of galaxies in the CF2 catalogue (left) compared to the 2MRS (right).

While the galaxy distribution is a biased tracer of the underlying mass density field of the dominant dark matter, the equivalence principle implies that galaxies are comoving with the dark matter on large scales away from non-gravitational forces. But the peculiar velocity field (as a function of the measured distance, $d_{e}$ ) derived from the noisy data suffers from inhomogeneous Malmquist biases (Lynden-Bell et al. 1988), resulting from the systematic difference between $d_{e}$ and the mean of true distances of galaxies with the same $d_{e}$. It is very difficult to correct for this bias because of its dependence on the unknown distribution of galaxies in true distance space. In contrast, galaxy biasing is likely to be well approximated by a simple linear relation $\delta_{g a l}=b \delta_{d m}$ between the galaxy and dark matter density fluctuations, as seen in Fig. 2.

In the standard paradigm, the observed structure has grown by Gravitational Instability (GI) from tiny initial fluctuations. Neglecting gas related effects, the equations of 


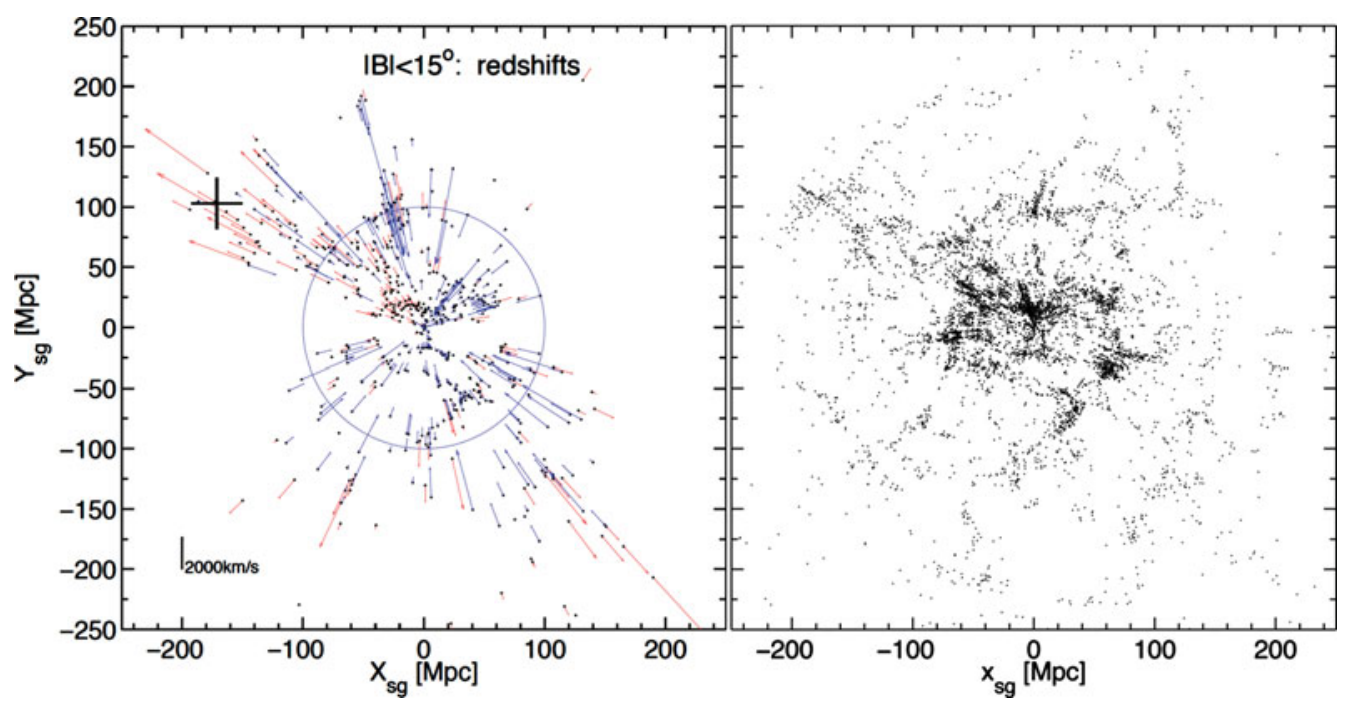

Figure 1. Left: The measured $v_{p}$ (in the CMB frame) of galaxies within $15^{\circ}$ of the SG plane in CF2. Black dots indicate the observed redshifts in the CMB frame. Red and blue arrows correspond to peculiar motions pointing away and towards the observer, respectively. The large cross plus sign indicates the location of the Shapley supercluster. Right: The distribution of galaxies within $20 \mathrm{Mpc}$ of the SG plane.

motion (EoM) of the perturbations are the usual Euler, Poisson and continuity equations in an expanding background. Supplemented with initial conditions appropriate for cosmological perturbations, the full solution to the EoM is possible only via numerical simulations which have achieved an impressive dynamical range from small galaxies to a significant fraction of the Hubble volume. Nonetheless, approximate solutions have been and will remain the basis for observational analyses methods and a physical understanding the numerical results. The simplest approximate solution is provided by linear theory which yields $\delta(\mathbf{x}, t)=\delta_{0}(\mathbf{x}) D+(t)+\delta_{-}(\mathbf{x}) D^{-}(t)$ where $D^{-}$describes a decaying mode and the growing mode obeys $\ddot{D}+2 H \dot{D}-3 \Omega H^{2} D / 2=0$. Linear theory also yields

$$
\delta=-\frac{1}{H f} \boldsymbol{\nabla} \cdot \mathbf{v}
$$

where $f=d \ln D / d \ln a \approx \Omega^{\gamma}$ is the growth rate and $\mathbf{v}(\mathbf{x})$ is the $3 \mathrm{D}$ peculiar velocity field. The index $\gamma$ depends on the cosmology (e.g. through the dark energy model) and the underlying theory for gravity. Accurate determination of $\gamma$ is one of the goals of future large surveys of galaxies. This $\delta-\mathbf{v}$ has been used extensively for the prediction of velocity fields associated with the distribution of galaxies in a given redshift survey. It is the basis for modeling redshift distortions of correlation functions from redshift surveys on large scales.

Within GI the two independent data sets can be analyzed in several ways: a) Correlation functions (and power spectra) have been estimated from the distribution of galaxies in redshift surveys. These correlations can be compared with predictions of cosmological models. Further, $c z=H d+v_{p}$ implies that correlations in redshift space indirectly probe $v_{p}$ through the fingers of god effect on small scales and the enhancement of clustering on large scales (Davis \& Peebles 1983; Kaiser 1988). b) Correlation analysis of the observed peculiar velocity field have also been done. However, this analysis is intrinsically plagued with inhomogeneous Malmquist biases. Quantitative conclusions from this type of analysis should always be examined critically. c) Comparison of low order moments of 


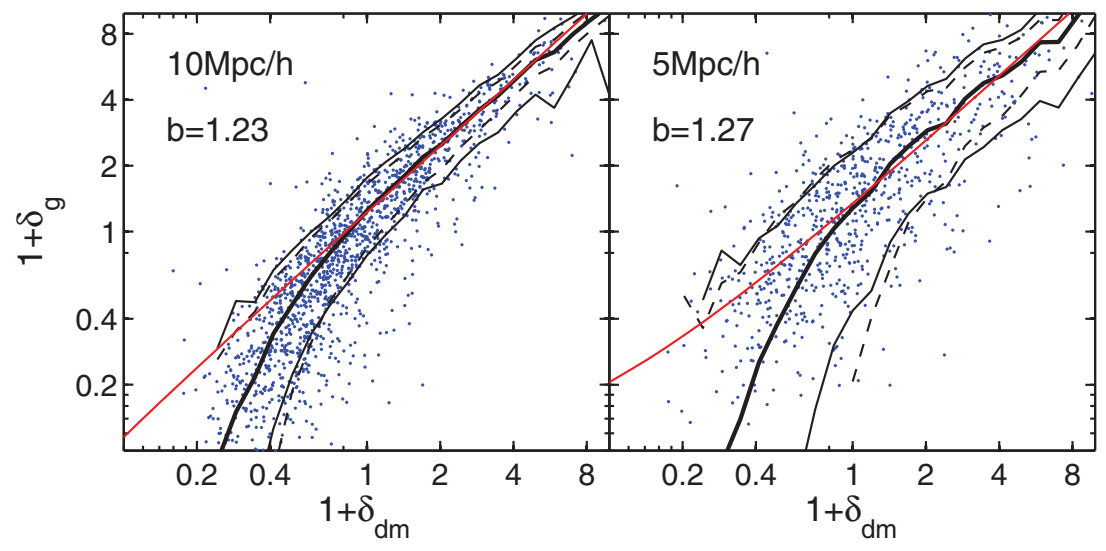

Figure 2. A scatter plot (logarithmic scale) of the galaxy versus the dark matter over-densities in 2MRS mock galaxy catalogs De Lucia \& Blaizot (2007). The left and right panels correspond to densities in cubic cells of $10 \mathrm{~h}^{-1} \mathrm{Mpc}$ and $5 \mathrm{~h}^{-1} \mathrm{Mpc}$ on the side, respectively. The thick solid curve in each panel is the mean of $1+\delta_{\mathrm{g}}$ at a given $1+\delta_{\mathrm{dm}}$. The two thin solid curves are $\pm 1 \sigma$ scatter computed from points above and below the mean. Dashed curves are the expected $\pm 1 \sigma$ Poisson (shot-noise) scatter. The nearly straight red lines show $\delta_{\mathrm{g}}=b \delta_{\mathrm{dm}}+$ const, where $b$ (indicated in the figure) are determined using linear regression from points in the range $-0.5<\delta_{\mathrm{dm}}<4$.

the peculiar velocity field, e.g. the bulk flow, with predictions of cosmological models. d) Testing GI by assessing the alignment of the gravitational force field (or the peculiar velocity) derived from redshift survey with the observed $v_{p}$ of galaxies in the peculiar velocity catalogs. This comparison is particularly important since it minimizes cosmic variance in the estimation of the cosmological parameters.

\section{The Zel'dovich approximation}

Full analytic solutions to the EoM are available only for initial conditions with a high degree of symmetry, e.g. self-similar collapse/expansion with planar, cylindrical or spherical symmetry. Zel'dovich (1970) proposed a remarkably simple approximation for the evolution of generic cosmological perturbations in the quasi-linear regime (laminar flow). The Zel'dovich approximation (ZA) states that the current position $\mathbf{x}(t)$ and the initial Lagrangian coordinate, $\mathbf{q}$, of a particle are related by

$$
\mathbf{x}=\mathbf{q}+D(t) \boldsymbol{\psi}(\mathbf{q}) .
$$

In the paper, Zel'dovich considered only baryons and argued that the natural perturbation scale is the Silk damping mass scale, $M_{\mathrm{S}} \approx 10^{12} M_{\odot}$. Further, the probability distribution of the eigenvalues of $\partial_{i} \psi_{j}$, revealed a preference for planar-like perturbations. Hence, ZA was the basis for the top-down pancake paradigm for structure formation. The approximation (2.1) is an exact solution to the full EoM for planar perturbations up to the onset of shell crossing (in collision-less fluids). The proof appears in Zeldovich \& Novikov (1983), but not in the 1970 paper. Although not highly accurate the ZA has given us fantastic physical insight into the working of nonlinear dynamics, e.g. the growth of angular momentum of galaxies (Doroshkevich 1970; White 1984), nonlinear density power spectrum with and without redshift distortions (Schneider \& Bartelmann 1995; Fisher \& Nusser 1996; Taylor \& Hamilton 1996; White 2014) and the probability distribution of the density field Kofman et al. (1994). 


\subsection{Extension: Lagrangian Perturbation Theory}

Here, the displacement is expanded in a Taylor series in an appropriate parameter (Buchert \& Ehlers 1993) which can be taken as the linear growth factor, $D$. Therefore, $\mathbf{x}=\mathbf{q}+\sum_{s} D^{s} \mathbf{\Psi}^{(s)}(\mathbf{q})$, where the ZA term $(s=1)$ is entirely fixed by the initial conditions, while the EoM dictate all $s>1$ terms via a recurrence relation involving lower order terms only (Zheligovsky \& Frisch 2014). Unfortunately these recurrence relations become messy for $s>2$. One of the reasons for that is the emergence of non-vanishing Lagrangian vorticity $\boldsymbol{\nabla}_{q} \times \boldsymbol{\Psi}^{(s)} \neq 0$ for $s>2 \dagger$. Second order Lagrangian perturbation $(2 \mathrm{LPT})(s=2)$, gives the density as

$$
\frac{1}{\rho_{2 \mathrm{LPT}}}=1+D \boldsymbol{\nabla} \cdot \mathbf{\Psi}^{(1)}+\frac{4}{7} D^{2}\left(\mu_{1} \mu_{2}+\mu_{1} \mu_{3}+\mu_{2} \mu_{3}\right) .
$$

where $\mu_{i}$ are the eigenvalues of $\partial_{i} \psi_{j}^{(1)}$. The continuity equation yields the widely known expression for the density in the ZA,

$$
\frac{1}{\rho_{\text {zel }}}=1+D \boldsymbol{\nabla} \cdot \Psi^{(1)}+D^{2}\left(\mu_{1} \mu_{2}+\mu_{1} \mu_{3}+\mu_{2} \mu_{3}\right)+D^{3} \mu_{1} \mu_{2} \mu_{3} .
$$

The ZA and 2LPT share the algebraic form of the second order term $\left(D^{2}\right)$, but with different coefficients. Thus, ZA is inaccurate even to second order. However, simulations show that The ZA provides a better match to the density derived from the velocity in (Gramann 1993) in high density regions, although 2LPT is better for low densities.

One of the most important common application of these approximation is the generation of particle displacements and velocities to be used as initial conditions for N-body simulations. The ZA and 2LPT are traditionally used but the latter yields is more accurate for this purpose.

\section{Peeble's action method}

We are given the positions of mass tracers (galaxies) today. What are the tracers' paths from the nearly homogeneous early Universe to the current configuration? This is a boundary value problem where a solution to the equations of motion is sought for boundary conditions (BC) at two different timesł. Its solution allows a reconstruction of the velocity field associate with the observed distribution of tracers and also the initial density field which lead to this distribution through gravitational interactions.

ZA and 2LPT can be employed to derive approximate solutions to the orbits. However, the most general (and elegant) method to do that has been designed by Peebles (1989) based on the least action principle. Orbits, $x(t)$, obeying the EoM also render the action stationary with respect to variations, $\delta x(t)$, satisfying the $\mathrm{BC} p \delta x=0$ at the limiting times $t_{1}$ and $t_{2}>t_{1}$, where $p$ is the momentum. In the cosmological problem, $\delta x\left(t_{2}\right)=0$ is naturally imposed. The initial positions are unknown, but Peebles noted that for the growing mode of cosmological perturbations the momentum vanishes as $t_{1} \rightarrow 0$. Hence, the solutions to the cosmological boundary value problem can be obtained by minimizing the action with respect to trial orbits constructed to satisfy a) known current positions and $b) p \rightarrow 0$ near the Big Bang. For sufficiently general trial functions the orbits should

$\dagger$ A vanishing Eulerian vorticity as a function of time (for an initial irrotational flow) is protected by Kelvin's circulation theorem until the onset of orbit-mixing. In other words, Eulerian vorticity remains zero for any order in perturbation theory.

$\ddagger$ It is closely tied to transport problems where displacements from a clumpy into a uniform distribution are sought by minimizing a cost function (Frisch et al. 2002). One still needs a dynamical prescription, e.g. ZA, to get the orbits from total displacements. 


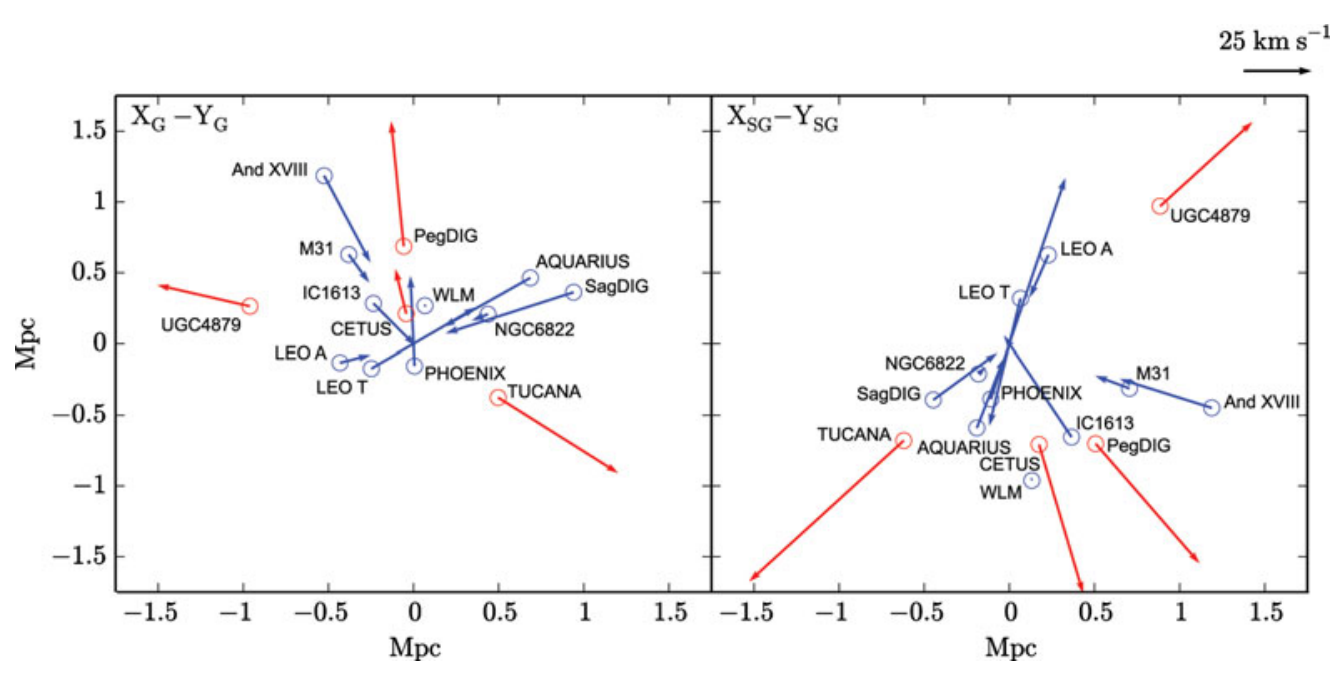

Figure 3. Galaxies in the LG in the Galactic (left) and SG (right) planes the arrow represent observed radial velocities transformed to the frame of reference comoving with the LG. Curtsey of Ziv Mikulsy.

be a solution to the full equations satisfying the BC, should such a solutions exist. In general, boundary value problems allow for multiple solutions or no solutions at all. Consider for example the linear oscillator $\ddot{x}+x=0$ subject to the $\mathrm{BC}, x\left(t_{1}=0\right)=0$ and $x\left(t_{2}=2 \pi\right)=0$. There is an infinite number of solutions: $x(t)=A \sin (t)$ for any $A$. There are also BC where the action has no extremum orbit. An example is $x(0)=0$ and $x(2 \pi)=1$. There is no physical orbit which satisfies these BC. In this case, it is easy to see that action can acquire infinitely large (positive and negative) values for certain choices of the orbits. For mixed BC where one of the conditions is $p=0$, the situation can even be more intriguing. The oscillator equation of motion constrained to $\dot{x}(0)=0$ and $x(2 \pi)=1$, is solved by $x(t)=\cos (t)$. Let us compute the action $S=\int_{0}^{2 \pi} d t\left(\dot{x}^{2}-x^{2}\right) / 2$ for the following choice for the perturbed orbits: $x_{p}=\cos (t)+A \cos (w t)$. These orbits satisfy the BC for $w=(2 n+1) / 4$, but not the equation of motion. It is easy to see that $S=A^{2} \pi\left(w^{2}-1\right) / 2$, i.e. the extremum point is a maximum for $w<1$ and a minimum for $w>1$.

\subsection{Application to the Local Group (LG) of galaxies: masses of $M W \mathscr{E} M 31$}

The LG contains about a dozen known galaxies within a distance $\sim 1.5 \mathrm{Mpc}$. Although not a virialized object (see below), it is gravitationally bound and detached from the expansion. Galaxy members (excluding satellites) of the LG are shown in Fig. 3. The MW and M31 are by far the most luminous (M31 is 100 more luminous than NGC6822the third most luminous galaxy shown in the figure). The radial velocities in the LG frame are represented by the arrow in the figure. The (radial) velocity dispersion is $\sim 60 \mathrm{kms}^{-1}$ and the flow pattern clearly reveals a non-virialized system that is most likely is on a first infall. Thus, the virial theorem will over-estimate the total mass of the LG. To constraint the masses of MW and M31, we apply the action principle to the nearby galaxies. The application is basically a generalization of the timing argue (TA) of Woltjer and Kahn who considered only the MW and M31. By treating the two galaxies as point particles with zero angular momentum (relative to the center of mass), TA constrains the total mass $M_{M W}+M_{31}$ by demanding that the two galaxies originated from zero separation a Hubble time ago, reaching their current separation and relative velocity today. The action 
method breaks the degeneracy between the masses by including kinematical observations of the smaller members of the LG. Although dynamically unimportant, the observed distances and velocities of these galaxies will allow us to resolve the individual masses $M_{M W}$ and $M_{31}$.

Galaxy orbits which render the action stationary are found iteratively using standard techniques. They are verified as solutions to the EoM in a leapfrog approximation. Since the solutions are non-unique, different choices of initial trial orbits will generally give different solutions for the galaxy paths. We define a $\chi^{2}$ measure of fit for all relevant observables, from which a best-fit solution can be selected. Maps of $\chi^{2}$ in four different scenarios, all with $H_{0}=67$ and $\Omega_{0}=0.27$, are shown in Fig. 4. At upper left are the contours in $\chi^{2}$ generated from a simplified catalog consisting of only MW and M31, to check the consistency of the action method with the Timing Argument. As expected, we find a well-defined constraint on the sum $M_{M W}+M_{M 31}$. The upper right panel, shows the results from a reduced version of our catalog which includes the LG actors but excludes external galaxies. The additional dynamical actors has broken the degeneracy in the TA, giving independent masses of $2.5 \pm 1.5 \times 10^{12} M_{\odot}$ for the $\mathrm{MW}$ and $3.5 \pm 1.0 \times 10^{12} M_{\odot}$ for M31. With the addition of the external galaxies (Fig. 4, lower left), the best mass for the MW increases to $3.5 \pm 1.0 \times 10^{12} M_{\odot}$. This is consistent at the lower end with previous TA measurements of the total LG mass and the individual MW mass. When the transverse velocity constraints on M31, LMC, M33, IC10, and LeoI are added (lower right), the confidence intervals are broadened and the best-fit mass for MW decreases slightly, to $3.0 \pm 1.5 \times 10^{12} M_{\odot}$, reflecting the fact that lower masses for MW are correlated to lower transverse velocities for M31 and other nearby galaxies. These values are to be understood as the masses contained within roughly half the separation between the two galaxies. For the MW, the value is more than twice what stellar motions yield for its virial mass. This could pose a challenge to the standard model since such an increase of the mass is not seen in N-body simulations.

\section{Cosmological constraints on 20-100 Mpc scales}

Restricting the analysis to these scales, away from non-linearities and hydrodynamical effects, greatly simplifies observational analyses of observations. Linear theory dynamical relations are, by and large, adequate on these scales but with the inclusion of scatter as a result from the presence of small scale modes. Further, large scale galaxy biasing is well described by a linear relation, as seen in Fig. 2.

\subsection{Velocity-velocity comparison}

Davis et al. (2011) have shown that GI passes a very important test: an excellent agreement between the velocity field predicted from the distribution of galaxies via linear theory and the observed motions of galaxies obtained from the TF measurements of spiral galaxies. The beauty of this test is that the effects of cosmic variance are minimized (in principles two good measurements are enough). The contribution of Marc Davis to these proceedings offers more details.

\subsection{The recovery of the $C M B$ dipole, i.e. the motion of the $L G$ with respect to the $C M B$}

The CMB temperature dipole together with astronomical estimation of the LG motion relative to the Sun, provide $V_{\mathrm{lg}}=627 \pm 22 \mathrm{~km} \mathrm{~s}^{-1}$ toward $(l, b)=\left(276^{\circ} \pm 3^{\circ}, 30^{\circ} \pm 3^{\circ}\right)$ for the LG motion relative to the CMB frame. The LG is accelerated by the cumulative gravitational pull of the surrounding large scale structure. Therefore, an important probe of the GI paradigm is whether the observed large scale structure, as traced by the galaxy 


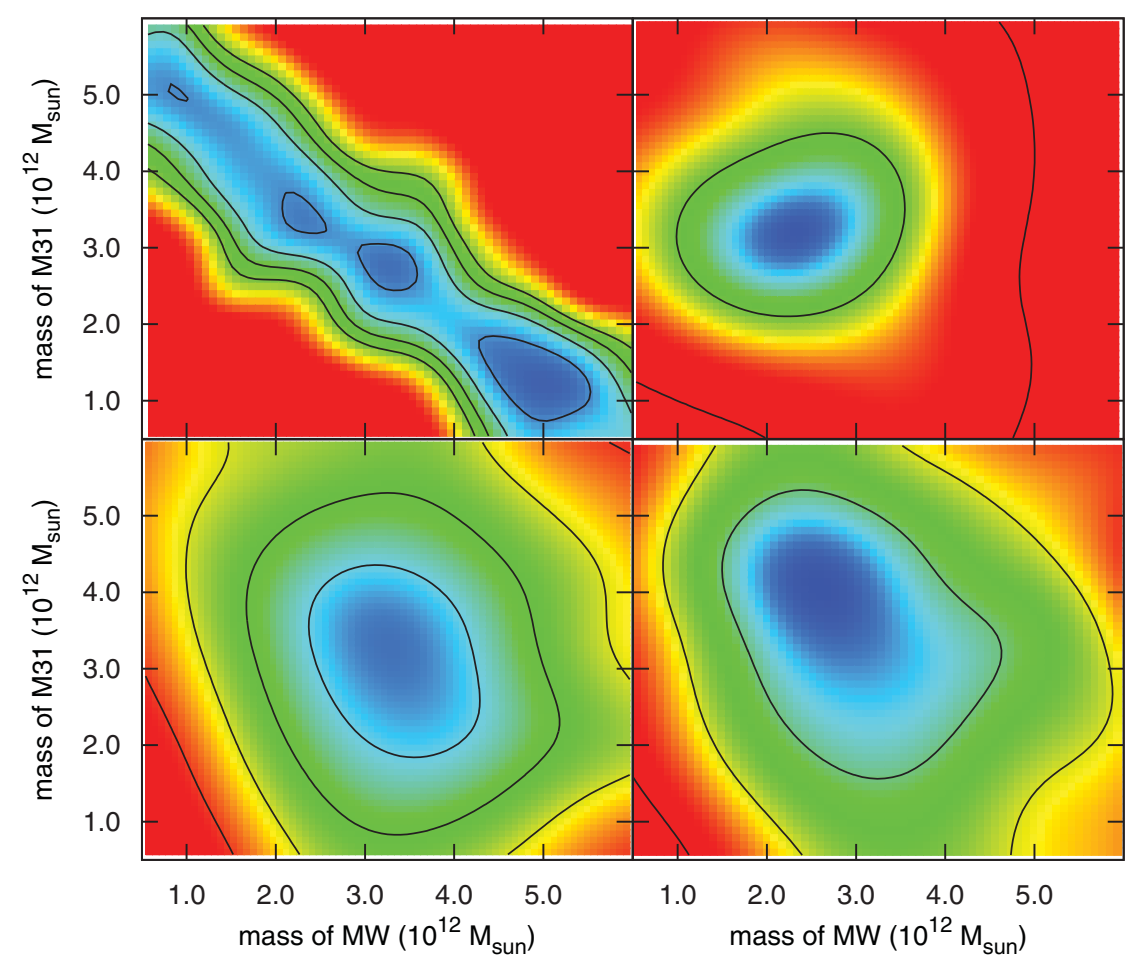

Figure 4. Contours in $\chi^{2}$ for different values of MW and M31 masses. Upper left: results from the two-body problem of MW + M31. Upper right: LG actors only. Lower left: LG actors + four external groups. Lower right: same as lower left, with transverse velocity constraints included for five nearby galaxies. The first contour level (solid black line) marks the region of $95 \%$ confidence. From Phelps et al. (2013).

distribution, could indeed account for the LG motion. To do that, we need to compute the gravitational force on the LG from an all sky survey of galaxies. According to linear theory, this force should be proportional to the LG motion. We use the 2MRS which is the deepest nearly-all sky survey of angular positions and spectroscopic galaxy redshifts limited to $K_{s}=11.75$ and arguably the best sample of objects to estimate the LG motion.

A source of uncertainty is the error in the linear theory dynamical reconstruction of the velocity from a given density field (equation 1.1). To assess this error we have estimated the LG motion from the full dark matter out to the largest possible outer radius in the simulation, i.e. $R_{\text {out }}=250 \mathrm{~h}^{-1} \mathrm{Mpc}$. The corresponding $1 \sigma$ error, $\sim 90 \mathrm{~km} \mathrm{~s}^{-1}$, is substantially smaller that the typical error in linear reconstruction of the peculiar velocity of a generic observer in the Universe. The reason for this is the strict criteria we have applied in selecting the "LG observer" in the mock catalogs, aimed at matching the quietness and moderate density environment of the observed LG. Removing these selection criteria boosts the error to $\gtrsim 300 \mathrm{~km} \mathrm{~s}^{-1}$, consistent with previous studies (Nusser $\&$ Branchini 2000). Nonlinear dynamical reconstruction methods can potentially reduce the dynamical error. However, because the particular environment of the LG, errors due to linear reconstruction are subdominant compared to the total error budget.

For a more realistic assessment of the recovery of the LG motion in real data we resort to mock catalogs designed to match the $2 \mathrm{MRS}$ with $K_{s}<11.75$ (as in Fig. 3). The results for various cases are shown in Fig. 5, as described in the caption. There is a dramatic decrease in the scatter when matter within $R_{\text {out }}=250 \mathrm{~h}^{-1} \mathrm{Mpc}$ is included. Still the 


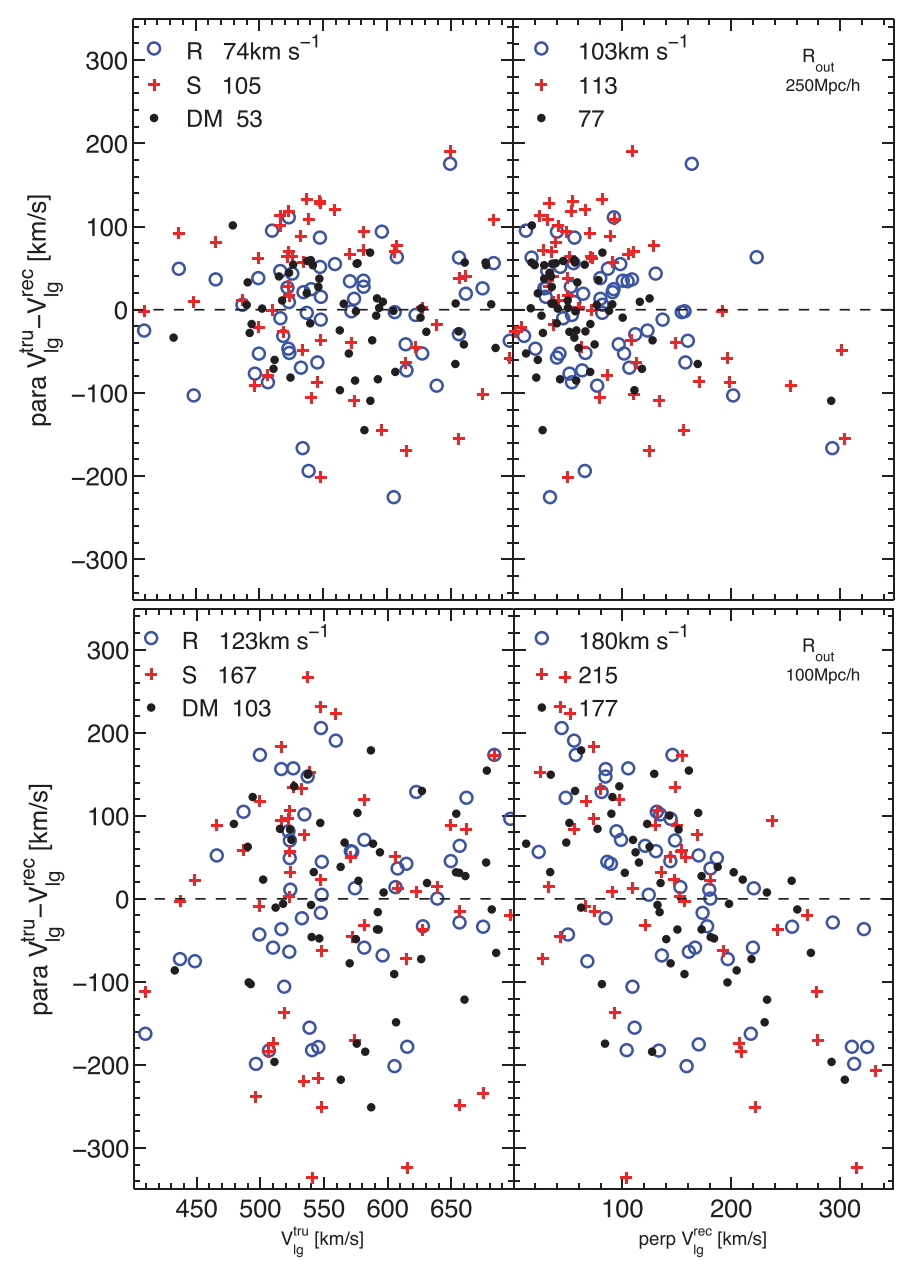

Figure 5. A scatter plot showing the velocity residual in the parallel and perpendicular directions from mock catalogs. The blue dots are in real space and the red crosses are in redshift space, while black dots show recovery from the full dark matter density field in real space. The rms values of the parallel and perpendicular residuals are listed in the left and right panels, respectively. Top and bottom panels correspond to velocity reconstruction with only data within $R_{\text {out }}=250 \mathrm{~h}^{-1} \mathrm{Mpc}$ and $R_{\text {out }}=100 \mathrm{~h}^{-1} \mathrm{Mpc}$, respectively. The rms of the parallel and perpendicular residuals are indicated, respectively, in the left and right panels.

residuals are at the level of $70-100 \mathrm{~km} \mathrm{~s}^{-1}$ depending on the case considered. This is consistent with Bilicki et al. (2011) who derived a similar result analytically. Current all sky data do not allow a reliable assessment of the contribution of fluctuations beyond $100 \mathrm{~h}^{-1} \mathrm{Mpc}$. Much of that is because of the Kaiser rocket effect (Kaiser 1987) (see Nusser et al. (2014) for further details).

\subsection{The bulk flow}

The bulk flow, $\mathbf{B}(r)$, is the mean motion of a sphere of a given radius, $r$. Computing $\mathbf{B}$ from the sparse and noisy peculiar velocity catalogs is very challenging. Improper handling of the data may easily lead to artificially large flow. Nusser \& Davis (2011) have estimated $\mathbf{B}(r)$ from the SFI++ survey. They have discarded $a$ ) the fainter galaxies which do not obey the TF relation and $b$ ) galaxies beyond $100 \mathrm{~h}^{-1} \mathrm{Mpc}$ which are 
likely to suffer from systematics related to the measurements of TF parameters. They found $\mathbf{B}(r)$ to be consistent with the standard $\Lambda$ CDM model and derived the constraint: $\sigma_{8}\left(\Omega_{m} / 0.266\right)^{0} .28=0.86 \pm 0.11$, which leads to a $\sigma_{8}$ higher (although consistent with) than the WMAP value. However, taking $\Omega_{m}=0.317$ from the recent Planck results, yields a best fit value $\sigma_{8}=0.819$, very close to the result reported by the Planck collaboration. Despite earlier claims of anomalous bulk flows, the emergent consensus is that bulk flow measurements from numerous data (intrinsic relations, $\mathrm{SN}$ and $\mathrm{kSZ}$ ), is consistent with the standard $\Lambda$ CDM model (Nusser \& Davis 2011; Dai et al. 2011; Colin et al. 2011; Hong et al. 2014; Feix et al. 2014; Watkins \& Feldman 2014; Ma \& Pan 2014; Planck Collaboration 2014).

\section{Summary}

The gravitational instability model for structure formation with its current $\Lambda$ CDM incarnation described the nearby large scale structure very well. Whatever corrections for this model should be small as far as large scales are concerned. Probing deviations from this model on large scales maybe possible only with next generation redshift surveys. Modifications on smaller scales $(\sim$ a few Mpcs) are a different matter (e.g. Peebles \& Nusser 2010) but are not the subject of this contribution.

Upcoming redshift surveys will contain a large number of galaxies to allow constraints on the cosmological velocity field independently of the classical redshift distortion the correlation functions. The idea is that galaxy redshifts $c z$ depend on $v_{p}$. Hence, using $c z$ instead of true distances, $d$, in order to estimate galaxy luminosities (from the observed apparent magnitudes) will introduce spatial variations of the luminosity function. Assuming negligible environmental dependence in the luminosity function, these variations can put constraints on the velocity field. (e.g. Yahil et al. 1980; Nusser et al. 2012a; Feix et al. 2014). Basically this method assumes that galaxy luminosity is a standard candle where the very large distance error is beaten by the large number of galaxies. The method can be applied to surveys with photometric redshifts (Bilicki et al. 2014) and is not restricted to spectroscopic surveys. The method can constrain galaxy biasing and the the linear growth rate is a velocity model based on the actual galaxy distribution is used to model the luminosity variations. The contribution of Martin Feix addresses potential caveats and presents an application to the SDSS.

Another potential probe is offered by Gaia. There could be a large number of galaxies detected as point sources by Gaia (Nusser et al. 2012b). For example, the nuclei of M87 and N5121 (both at $\mathrm{d}=17.8 \mathrm{Mpc}$ ) should be detected with an end of mission accuracy of $600 \mathrm{~km} \mathrm{~s}^{-1}$ in the transverse motion. The surface brightness profiles of the Carnegie-Irvine Galaxy Survery show that $70 \%$ of galaxies in this survey could be detected by Gaia. The majority of these nearby galaxies will be detected if placed at $\gtrsim 500 \mathrm{Mpc}$ (early type) and $\gtrsim 250 \mathrm{Mpc}$ (late type). Of course, parallax distance errors increase quadratically with distance. Therefore, the error on Gaia's distances for extragalactic objects will be huge and cannot be used to get the transverse velocities from the measured proper motions. But, at such distances, the redshifts can be used as proxies for the true distance without introducing a significant error in the transverse velocities.

\section{References}

Bilicki, M., Chodorowski, M., Jarrett, T., \& Mamon, G. A. 2011, ApJ, 741, 31

Bilicki, M., Jarrett, T. H., Peacock, J. A., Cluver, M. E., \& Steward, L. 2014, ApJ. S, 210, 9

Buchert, T. \& Ehlers, J. 1993, MNRAS, 264, 375 
Colin, J., Mohayaee, R., Sarkar, S., \& Shafieloo, A. 2011, MNRAS, 414, 264

Dai, D., Kinney, W. H., \& Stojkovic, D. 2011, ArXiv:1102.0800

Davis, M., Nusser, A., Masters, K. L., Springob, C., Huchra, J. P., \& Lemson, G. 2011, MNRAS, 413, 2906

Davis, M. \& Peebles, P. J. E. 1983, ApJ, 267, 465

De Lucia, G. \& Blaizot, J. 2007, MNRAS, 375, 2

Doroshkevich, A. G. 1970, Astrophysics, 6, 320

Feix, M., Nusser, A., \& Branchini, E. 2014, JCAP, 9, 19

Fisher, K. B. \& Nusser, A. 1996, MNRAS, 279, L1

Frisch, U., Matarrese, S., Mohayaee, R., \& Sobolevski, A. 2002, Nature, 417, 260

Gramann, M. 1993, ApJ, 405, 449

Hong, T., Springob, C. M., Staveley-Smith, L. et al. 2014, MNRAS, 445, 402

Huchra, J. P., Macri, L. M., Masters, K. L. et al. 2012, ApJ. S, 199, 26

Kaiser, N. 1987, MNRAS, 227, 1

一. 1988, MNRAS, 231, 149

Kofman, L., Bertschinger, E., Gelb, J. M., Nusser, A., \& Dekel, A. 1994, ApJ, 420, 44

Lynden-Bell, D., Faber, S. M., Burstein, D. et al. 1988, ApJ, 326, 19

Ma, Y.-Z. \& Pan, J. 2014, MNRAS, 437, 1996

Masters, K. L., Springob, C. M., Haynes, M. P., \& Giovanelli, R. 2006, ApJ, 653, 861

Nusser, A. \& Branchini, E. 2000, MNRAS, 313, 587

Nusser, A., Branchini, E., \& Davis, M. 2012a, ApJ, 744, 193

—. 2012b, ApJ, 755, 58

Nusser, A. \& Davis, M. 2011, ApJ, 736, 93

Nusser, A., Davis, M., \& Branchini, E. 2014, ApJ, 788, 157

Peebles, P. J. E. 1980, The large-scale structure of the universe (Princeton University Press)

一. 1989, ApJL, 344, L53

Peebles, P. J. E. \& Nusser, A. 2010, Nature, 465, 565

Phelps, S., Nusser, A., \& Desjacques, V. 2013, ApJ, 775, 102

Planck Collaboration. 2014, A\&BA, 561, A97

Schneider, P. \& Bartelmann, M. 1995, MNRAS, 273, 475

Taylor, A. N. \& Hamilton, A. J. S. 1996, MNRAS, 282, 767

Tully, R. B. et al. 2013, Astronomical. J, 146, 86

Watkins, R. \& Feldman, H. A. 2014, ArXiv e-prints

White, M. 2014, MNRAS, 439, 3630

White, S. D. M. 1984, ApJ, 286, 38

Yahil, A., Sandage, A., \& Tammann, G. A. 1980, ApJ, 242, 448

Zeldovich, I. B. \& Novikov, I. D. 1983, Relativistic astrophysics. Volume 2 - The structure and evolution of the universe (Chicago, IL, University of Chicago Press, 1983)

Zel'dovich, Y. B. 1970, $A \mathscr{E} A$ A, 5, 84

Zheligovsky, V. \& Frisch, U. 2014, Journal of Fluid Mechanics, 749, 404 